\title{
BMJ Open Efficacy of interventions to improve physical activity levels in individuals with stroke: a systematic review protocol
}

\author{
Larissa Tavares Aguiar, ${ }^{1,2}$ Júlia Caetano Martins, ${ }^{1}$ Sylvie Nadeau, ${ }^{2,3}$ \\ Raquel Rodrigues Britto, ${ }^{1}$ Luci F Teixeira-Salmela, ${ }^{1}$ Christina D C M Faria ${ }^{1}$
}

To cite: Aguiar LT,

Martins JC, Nadeau S, et al. Efficacy of interventions to improve physical activity levels in individuals with stroke: a systematic review protocol. BMJ Open 2017;7 e012479. doi:10.1136/ bmjopen-2016-012479

- Prepublication history and additional material is available. To view please visit the journal (http://dx.doi.org/ 10.1136/bmjopen-2016012479).

Received 29 April 2016 Revised 19 September 2016 Accepted 20 September 2016

\section{CrossMark}

For numbered affiliations see end of article.

\section{Correspondence to} Dr Christina DCM Faria; cdcmf@ufmg.br, chrismoraisf@gmail.com

\section{ABSTRACT}

Introduction: Stroke is a leading health problem worldwide and an important cause of disability. Stroke survivors show low levels of physical activity, and increases in physical activity levels may improve function and health status. Therefore, the aims are to identify which interventions that have been employed to increase physical activity levels with stroke survivors, to verify their efficacy and to identify the gaps in the literature.

Methods and analysis: A systematic review of randomised controlled trials that investigated the efficacy of interventions aiming at increasing physical activity levels of stroke survivors will be conducted. Electronic searches will be performed in the MEDLINE, Physiotherapy Evidence Database (PEDro), Excerpta Medica (EMBASE), Literatura Latino-Americana e do Caribe em Ciências da Saúde (LILACS) and Scientific Electronic Library Online (SCIELO) databases. Hand searches of the reference lists of the included studies or relevant reviews will also be employed. Two independent reviewers will screen all the retrieved titles, abstracts and full texts. A third reviewer will be referred to solve any disagreements. The quality of the included studies will be assessed by the PEDro Rating Scale. This systematic review will also include a qualitative synthesis. Meta-analyses will be performed, if the studies are sufficiently homogeneous. This review will follow the Preferred Reporting Items for Systematic Review and Meta-Analysis (PRISMA) statement. The quality of the evidence regarding physical activity will be assessed, according to the Grading of Recommendations Assessment, Development and Evaluation (GRADE).

Discussion: This systematic review will provide information on which interventions are effective for increasing physical activity levels of stroke survivors. This evidence may be important for clinical decisionmaking and will allow the identification of gaps in the literature that may be useful for the definition of future research goals and the planning of new trials.

Trial registration number: CRD42016037750.

\section{INTRODUCTION}

Stroke is a leading health problem worldwide and an important cause of long-term

\section{Strengths and limitations of this study}

- This systematic review protocol will be reported in accordance with the Preferred Reporting Items for Systematic Review and Meta-Analysis (PRISMA) statement.

- The three most complete databases for reports of randomised controlled trials will be searched.

- The quality of the included studies will be assessed by the PEDro Rating Scale.

- The quality of the evidence will be assessed, based on the Grading of Recommendations Assessment, Development and Evaluation (GRADE).

- This systematic review will not include interventions based on invasive procedures, drug and nutrition therapies, neither other neurological populations besides stroke.

disabilities. ${ }^{1}{ }^{2}$ Although stroke mortality rate has decreased, the majority of the growth of the global stroke burden is coming from developing countries and it is expected to increase as a result of demographic changes, such the increases in the ageing population. ${ }^{1}{ }^{2}$ Stroke survivors are more likely to require help with activities and to have restrictions in participation than matched controls. ${ }^{3}$ In addition, stroke survivors are at higher risks of having other cardiovascular diseases (CVDs).$^{2}$ Therefore, it is essential to develop interventions to recover and promote health and function, as well as to prevent secondary diseases in poststroke survivors.

Physical activity is defined as any bodily movement produced by skeletal muscles that result in energy expenditure, such as those performed during activities of daily living, at work, at home, during leisure activities or transport. ${ }^{4}$ Stroke survivors have low levels of physical activity at hospital and community settings. ${ }^{5}{ }^{6}$ The quantity, duration and intensity of physical activities are reduced, even in high functioning community-dwelling stroke 
survivors, when matched with a healthy elderly. ${ }^{7}$ According to a population-based study, communitydwelling stroke survivors have the highest proportion of physical inactivity, when compared with older adults with diabetes, musculoskeletal, cardiovascular, respiratory or other neurological chronic diseases. ${ }^{8}$

One of the major consequences of the disabilities after stroke is a chronic sedentary lifestyle. ${ }^{9}$ Common disabilities observed after stroke, including muscular weakness ${ }^{10}$ reduced cardiorespiratory fitness, ${ }^{11}$ fatigue ${ }^{12}$ physical mobility limitations, ${ }^{13}{ }^{14}$ low perceptions of quality of life ${ }^{15}$ and restrictions in social participation, ${ }^{3}$ may lead to low physical activity lifestyles. ${ }^{9}$ Low levels of physical activity, in turn, have a negative impact on these disabilities and are related to health problems and, therefore, create a vicious cycle. ${ }^{9}$

Increase in physical activity levels can improve function and health in individuals after stroke. $^{9}$ Furthermore, increases in physical activity levels could reduce the recurrence of stroke and other CVDs. ${ }^{16}$ Recently, the American Heart Association and the American Stroke Association published a scientific statement with recommendations of physical activity for stroke survivors, ${ }^{9}$ and there is a consensus that increases in physical activity levels are important for public health systems worldwide. ${ }^{9}{ }^{17}$ However, according to the best of our knowledge, no broad systematic reviews on this topic have been conducted. There was found only two specific systematic reviews that investigated the efficacy of interventions on physical activity levels in stroke survivors: one targeted behavioural change ${ }^{18}$ and the other selfmanagement programmes. ${ }^{19}$ The results of both reviews showed, in general, improvements in physical activity levels after tailored counselling ${ }^{18}$ and self-management programmes. ${ }^{19}$ Nevertheless, the risk of bias in the included studies was high in both reviews. ${ }^{18} 19$ Therefore, the overall efficacy of those interventions to improve physical activity levels in stroke survivors remains uncertain. In addition, these reviews had strict eligibility criteria, which may have prevented the inclusion of other relevant studies. ${ }^{18} 19$ For instance, they included studies that had follow-up measures at 3 months or longer ${ }^{18}$ and that only included community-dwelling participants. ${ }^{19}$ Therefore, the aims of the present systematic review are to identify which interventions have been employed to increase physical activity levels and to verify the efficacy of these interventions in individuals with stroke. The ultimate goal is to identify the gaps in the literature to allow for the planning and development of new clinical trials.

\section{METHODS AND ANALYSES}

\section{Study design}

This systematic review protocol will be reported in accordance with the Preferred Reporting Items for Systematic review and Meta-Analysis Protocols (PRISMA-P) (see Research Checklist), ${ }^{20} 21$ and the results will be reported following the Preferred Reporting Items for Systematic Review and Meta-Analysis (PRISMA) statement. ${ }^{22} 23$

\section{Study registration}

On the basis of the PRISMA-P guidelines, this systematic review protocol was registered with the International Prospective Register of Systematic Reviews (PROSPERO) on 14 April 2016 (registration number: CRD42016037750; http://www.crd.york.ac.uk/PROSPERO/).

\section{Eligibility criteria}

\section{Types of study}

All randomised controlled trials (RCTs) that investigated the efficacy of interventions aiming at increasing physical activity levels in stroke survivors will be included in this systematic review. Quasi-RCTs, controlled clinical trials, cross-sectional studies, case series and case reports will be excluded.

\section{Participants}

All RCTs, in which participants were adults ( $\geq 18$ years of age) and survived a stroke, will be included, without further restrictions. The authors of the studies that included mixed groups will be contacted for specific data related to the stroke survivors. When specific data are not available, the study will be excluded. Studies with participants with transient ischaemic attack will also not be included.

\section{Types of interventions}

All RCT that employed any type and mode of delivery, including, but not limited to, aerobic, strength exercises, counselling, self-management or behavioural interventions, in isolation or in combination, aimed at increasing physical activity levels, will be included. Trials will be excluded if the experimental interventions were invasive procedures, drug and nutrition therapies.

\section{Comparisons or control}

No restrictions will be made on the comparisons and/or the control group.

\section{Outcome measures}

Studies that quantified physical activity levels by any method, such as self-report assessment tools (eg, selfreport questionnaires, diaries/logs or recall interviews ${ }^{24}$ or by direct measures (eg, accelerometers, pedometers, doubly labeled water, multisensor tools or direct observations) will be included. ${ }^{24}{ }^{25}$ Physical activity levels could be reported as energy expenditure, steps per day, time of physical activity per day, number of transitions, time spent upright or others. Trials reporting walking or exercise capacity, gait patterns or ability to perform activities of daily living (eg, Barthel or Functional Independence Measure scores), which are not measurements of physical activity levels, will be excluded. Studies reporting only sedentary time will also be excluded. 
Search strategy for the identification of relevant studies Electronic searches will be conducted in the MEDLINE (via PubMed), Physiotherapy Evidence Database (PEDro), Excerpta Medica (EMBASE), Literatura Latino-Americana e do Caribe em Ciências da Saúde (LILACS) and Scientific Electronic Library Online (SCIELO) databases, from their inception to February 2016, without any language restrictions. The MEDLINE, PEDro and EMBASE databases were chosen because they are the most complete databases for reports of RCT. $^{26}$ The LILACS and SCIELO databases were chosen because they contain articles published in the Portuguese or Spanish languages. Hand searches of the reference lists of the included studies or identified relevant reviews will be employed. The search strategy will include terms related to stroke, RCT and physical activity levels. The search strategy related to stroke and RCT, which will be employed at the MEDLINE database, will follow that of a recent Cochrane Systematic Review ${ }^{27}$ and the Cochrane Handbook for Systematic Reviews of Interventions, ${ }^{28}$ respectively. The search strategy related to physical activity levels will be a combination of terms employed on three previously published systematic reviews. ${ }^{24} 2930$ See online supplementary file 1 for the MEDLINE full-search strategy. The search strategy for the MEDLINE will be adapted to suit the other databases.

\section{Screening of the studies}

Duplicate studies will be removed. The main author (LTA) will search all databases and extract the titles and abstracts. Two independent reviewers (LTA, JCM) will screen all the retrieved titles and abstracts from the electronic search, according to the previously described inclusion criteria. Full texts will be screened by the same reviewers (LTA, JCM), independently. A third reviewer (CDCMF) will be referred to solve any disagreements. All the reasons for exclusion of ineligible studies will be recorded. The results of the screening process will be provided in details using the PRISMA information flow. $^{21}$

\section{Data extraction}

The two independent reviewers (LTA, JCM) will extract the data, following recommended guidelines. Data extraction will include: (1) study details: authors and year of publication; (2) study characteristics: inclusion/ exclusion criteria and setting; (3) sample characteristics: number of participants, age, sex, type and time since the onset of the stroke; (4) methods: design and allocation, blinding, sampling, time points when data were collected, loss to follow-up, recruitment and retention rates, comparison/control group; (5) interventions: description of intervention, duration, frequency, intensity, length and supervision and (6) outcomes: description, measurement instruments, unit of measurement and intervention effects on the outcome. Any additional information that may express conflict of interest or bias will also be extracted. The correspondening author of the studies with missing or incomplete data will be contacted for further information. Disagreements will be discussed with the third reviewer (CDCMF).

\section{Risk of bias}

The quality of the included RCT will be assessed by extracting the PEDro scores from the PEDro database (http://www.pedro.org.au). The PEDro Rating Scale is an 11-item checklist, which gives scores that range from 0 to 10 , designed for rating the methodological quality of trials. The RCTs, which have not been assessed by the PEDro rating scale, will be scored by the reviewers (LTA, JCM). Once again, disagreements will be discussed with the third reviewer (CDCMF). The scores on the individual items of the PEDro scale of all included trial will be reported in a table. In an attempt to determine if reporting bias is present in the included trials, the trial register's 'ClinicalTrials.gov', 'http://www.anzctr.org.au' and 'http://www.clinicaltrialsregister.eu/ctr-search/search' will be screened to assess whether selective reporting is present.

\section{Quality of evidence}

The quality of the evidence of the studies will be assessed based on the Grading of Recommendations Assessment, Development and Evaluation (GRADE). ${ }^{31}$ The quality of the studies will be judged as high (further research is very unlikely to change the confidence in the effect estimates), moderate (further research is likely to have an important impact on the confidence in the effect and may change the estimate), low (further research is very likely to have an important impact on the confidence in the effect and is likely to change the estimate) and very low (any estimate of the effect is very uncertain). ${ }^{31}$

\section{Strategy for data synthesis}

This systematic review will also include a qualitative synthesis, which will provide information, in text and tables, to summarise the results of the included studies. A narrative synthesis will be performed to explore the results and associations within and between the included trials. Forest-plots and meta-analyses will be conducted if the studies are sufficiently homogeneous regarding the interventions and outcomes and if sufficient data are available, to synthesise the direction, size and consistency of the possible effects, using the Review Manager software (RevMan) (Review Manager (RevMan) [Computer program]. Version 5.3. Copenhagen: The Nordic Cochrane Centre, The Cochrane Collaboration, 2014).

\section{Analyses of subgroups or subsets}

If sufficient data are available, subgroups analyses will be carried out. These analyses will assess differences between the stroke phases (eg, acute, subacute or chronic), physical activity measurements (eg, subjective 
vs objective instruments), type, duration and delivery of the intervention, group comparisons, quality and risk of bias.

\section{DISCUSSION}

According to the best of our knowledge, this systematic review is the first to investigate the efficacy of broad types of interventions aimed at increasing physical activity levels in stroke survivors. Previous systematic reviews with the aim to examine the efficacy of interventions on physical activity levels in poststroke survivors have investigated only two specific types of interventions: targeted behavioural change ${ }^{18}$ and self-management programmes. ${ }^{19}$ However, the efficacy of those interventions to increase physical activity levels in poststroke survivors continue to be unclear, due to the fact that the risk of bias of the included trials was high in both reviews. ${ }^{18} 19$ Moreover, important trials might not have been included because of the stringent eligibility criteria of these reviews. ${ }^{18} 19$ These factors limit the interpretation of the findings regarding the impacts of interventions on physical activity levels in poststroke survivors. Considering that physical inactivity is a major risk for recurrence of stroke and other CVDs and may affect health and function, ${ }^{2} 916$ it is important to investigate the impact of different types of interventions on physical activity levels in poststroke survivors.

The results of this systematic review will provide comprehensive and rigorous evidence regarding which types of interventions, and/or specific protocols have been investigated and are effective for increasing physical activity levels of stroke survivors. The information from the qualitative synthesis, which will be developed to explore the results and relations within and between the included studies, will be important for clinical decisionmaking aiming at improving function and health status of stroke survivors. Moreover, if sufficiently homogeneous data to conduct meta-analyses are available, clinicians will have information regarding the expected effect size associated with a given intervention.

Furthermore, this systematic review may allow the identification of gaps in the literature, regarding the types and specific intervention protocols, group comparisons, measurement instruments, short-term and longterm effects and different stroke phases (eg, acute, subacute or chronic). This information will be useful for the definition of future research goals and the planning of new research trials. The results from this systematic review will be spread by scientific publication and presentations in scientific events.

\section{Author affiliations}

${ }^{1}$ Department of Physical Therapy, Universidade Federal de Minas Gerais (UFMG), Belo Horizonte, Brazil

2École de réadaptation, Université de Montréal (UdeM), Montréal, Quebec, Canada

${ }^{3}$ Centre de recherche interdisciplinaire en réadaptation (CRIR), Institut de réadaptation Gingras-Lindsay de Montréal (IRGLM), CIUSSS Centre-Sud-del'Île-de-Montréal, Montréal, Quebec, Canada
Contributors The guarantor of this study is CDCMF. All authors contributed to the conception and design of the study, development of the search strategy, the establishment of the inclusion and exclusion criteria, data extraction criteria, analyses and interpretation. LTA and JCM were involved in the data collection and writing. CDCMF, SN, RRB and LFT-S provided critical revision of the paper. All authors read and provided final approval of the version to be published.

Funding Financial support for this research was provided by Coordenação de Aperfeiçoamento de Pessoal de Nível Superior (CAPES), Fundação de Amparo à Pesquisa do Estado de Minas Gerais (FAPEMIG), Conselho Nacional de Desenvolvimento Científico e Tecnológico (CNPq) and Pró-reitoria de Pesquisa da Universidade Federal de Minas Gerais (PRPq/UFMG). This financial support provides scholarships and grants. CAPES, FAPEMIG, CNPq and PRPq/UFMG are not involved in any other aspect of this study protocol.

Competing interests None declared.

Provenance and peer review Not commissioned; externally peer reviewed.

Data sharing statement We, authors, agree that, should the article be accepted, the BMJ Open shall take over the authors' rights relating to this article, which shall become the property of the Journal.

Protocol amendments Protocol amendments will be documented with the date of each amendment and with a description of the change and the rationale.

Open Access This is an Open Access article distributed in accordance with the Creative Commons Attribution Non Commercial (CC BY-NC 4.0) license, which permits others to distribute, remix, adapt, build upon this work noncommercially, and license their derivative works on different terms, provided the original work is properly cited and the use is non-commercial. See: http:// creativecommons.org/licenses/by-nc/4.0/

\section{REFERENCES}

1. Feigin VL, Krishnamurthi RV, Parmar $P$, et al. Update on the global burden of ischemic and hemorrhagic stroke in 1990-2013: the GBD 2013 study. Neuroepidemiology 2015;45:161-76.

2. Mozaffarian D, Benjamin EJ, Go AS, et al. Heart disease and stroke statistics-2016 update: a report from the American Heart Association. Circulation 2016;133:e38-360.

3. Skolarus LE, Burke JF, Brown DL, et al. Understanding stroke survivorship: expanding the concept of poststroke disability. Stroke 2014;45:224-30.

4. Caspersen CJ, Powell KE, Christenson GM. Physical activity, exercise, and physical fitness: definitions and distinctions for health-related research. Public Health Rep 1985;100:126-31.

5. West T, Bernhardt J. Physical activity in hospitalised stroke patients. Stroke Res Treat 2012;2012:813765.

6. English C, Manns PJ, Tucak C, et al. Physical activity and sedentary behaviors in people with stroke living in the community: a systematic review. Phys Ther 2014;94:185-96.

7. Field MJ, Gebruers N, Shanmuga ST, et al. Physical activity after stroke: a systematic review and meta-analyses. ISRN Stroke 2013;2013:1-13.

8. Ashe MC, Miller WC, Eng JJ, et al. Older adults, chronic disease and leisure-time physical activity. Gerontology 2009;55:64-72.

9. Billinger SA, Arena R, Bernhardt J, et al. Physical activity and exercise recommendations for stroke survivors: a statement for healthcare professionals from the American Heart Association/ American Stroke Association. Stroke 2014;45:2532-53.

10. Hill TR, Gjellesvik TI, Moen PMR, et al. Maximal strength training enhances strength and functional performance in chronic stroke survivors. Am J Phys Med Rehabil 2012;91:393-400.

11. Marsden DL, Dunn A, Callister R, et al. Characteristics of exercise training interventions to improve cardiorespiratory fitness after stroke a systematic review with meta-analyses. Neurorehabil Neural Repair 2013;27:775-88.

12. Jones TM, Dean CM, Dear BF, et al. An internet survey of the characteristics and physical activity of community-dwelling Australian adults with acquired brain injury: exploring interest in an internet-delivered self-management program focused on physical activity. Disabil Health J 2016;9:54-63.

13. IJmker T, Houdijk H, Lamoth CJ, et al. Effect of balance support on the energy cost of walking after stroke. Arch Phys Med Rehabil 2013;94:2255-61. 
14. Faria CD, Teixeira-Salmela LF, Nadeau S. Predicting levels of basic functional mobility, as assessed by the Timed "Up and Go" test, for individuals with stroke: discriminant analyses. Disabil Rehabil 2013;35:146-52.

15. Polese JC, Pinheiro MB; Machado GC, et al. Chronic hemiparetic subjects with higher physical activity levels report better quality of life. Rev Neurociênc 2014;22:221-6.

16. Walter NK, Bruce O, Henry RB, et al. On behalf of the American Heart Association Stroke Council, Council on Cardiovascular and Stroke Nursing, Council on Clinical Cardiology, and Council on Peripheral Vascular Disease. Guidelines for the Prevention of Stroke in Patients With Stroke and Transient Ischemic Attack A Guideline for Healthcare Professionals From the American Heart Association/ American Stroke Association. Stroke 2014;45:2160-236.

17. Fernhall B, Borghi-Silva A, Babu AS. The future of physical activity research: funding, opportunities and challenges. Prog Cardiovasc Dis 2015;57:299-305.

18. Morris $\mathrm{JH}$, Macgillivray S, McFarlane S. Interventions to promote long-term participation in physical activity after stroke: a systematic review of the literature. Arch Phys Med Rehabil 2014;95:956-67.

19. Jones TM, Dean CM, Hush JM, et al. A systematic review of the efficacy of self-management programs for increasing physical activity in community-dwelling adults with acquired brain injury (ABI). Syst Rev 2015;4:51.

20. Moher D, Shamseer L, Clarke M, et al., The Future of Physical Activity Research: Funding, Opportunities and Challenges; PRISMA-P Group. Preferred reporting items for systematic review and meta-analyses protocols (PRISMA-P) 2015 statement. Syst Rev 2015;4:1.

21. Shamseer L, Moher D, Clarke M, et al., PRISMA-P Group. Preferred reporting items for systematic review and meta-analyses protocols (PRISMA-P) 2015: elaboration and explanation. BMJ 2015;349:97647.

22. Moher D, Liberati A, Tetzlaff J, et al., The PRISMA Group. Preferred reporting items for systematic reviews and meta-analyses: the PRISMA statement. Ann Intern Med 2009;151:264-9.
23. Liberati A, Altman DG, Tetzlaff J, et al. The PRISMA statement for reporting systematic reviews and meta-analyses of studies that evaluate health care interventions: explanation and elaboration. PLoS Med 2009;6:e1000100.

24. Strath SJ, Kaminsky LA, Ainsworth BE, et al. Guide to the assessment of physical activity: clinical and research applications: a scientific statement from the American Heart Association. Circulation 2013;128:2259-79.

25. Fini NA, Holland AE, Keating J, et al. How is physical activity monitored in people following stroke? Disabil Rehabil 2014;6: $1-15$.

26. Michaleff ZA, Costa LO, Moseley AM, et al. CENTRAL, PEDro, PubMed, and EMBASE are the most comprehensive databases indexing randomized controlled trials of physical therapy interventions. Phys Ther 2011;91:190-7.

27. Barclay RE, Stevenson TJ, Poluha W, et al. Interventions for improving community ambulation in individuals with stroke. Cochrane Database Syst Rev 2015;3:CD010200.

28. Higgins JPT, Green S, eds. Cochrane handbook for systematic reviews of interventions, Version 5.1.0 (updated March 2011). The Cochrane Collaboration, 2011. http://www.cochrane-handbook.org (accessed 14 Apr 2016).

29. Vries $\mathrm{H}$, Kooijman $\mathrm{T}$, Ittersum $\mathrm{M}$, et al. Does an activity monitor based intervention increase daily physical activity of adults with overweight or obesity? A systematic review and meta-analyses. PROSPERO 2015:CRD42015024086. http://www.crd.york.ac.uk/ PROSPERO/display_record.asp?ID=CRD42015024086 (accessed 14 Apr 2016)

30. Richards J, Hillsdon M, Thorogood M, et al. Face-to-face interventions for promoting physical activity. Cochrane Database Syst Rev 2013;9:CD010392.

31. Guyatt GH, Oxman AD, Vist GE, et al., GRADE Working Group. GRADE: an emerging consensus on rating quality of evidence and strength of recommendations. BMJ 2008;336:924-6. 